\title{
Hematomas bulbares puros
}

\author{
M. Jato, F. Rubio
}

PURELY BULBAR HEMATOMAS

Summary. Introduction. Purely bulbar hematomas are not common (0.25\%-0.50\% of all intraparenchymatous hematomas), probably because of the particular characteristics of the anatomy and hemodynamics of the bulbar circulation. Clinical cases. We selected 18 cases. Three of these were our own and the purely bulbar localization was confirmed by neuroimaging surgical findings and necropsy. Its appearance was related to arterial hypertension, anticoagulant treatment and obvious or occult vascular malformations, although in a considerable proportion (39\%) no cause was found. Although arterial hypertension is less important in its etiopathogenesis as compared with other sites of intraparenchymatous hemorrhage, 7 of the patients were hypertensive. The commonest clinical findings were nystagmus (72\%), and dysphagia and hypoglossal paralysis (61\%). The commonest site was posterior. Arteriography was of little use since this was normal in all the cases in which it was done (72\%). The presence of angiographically occult malformations made it advisable to use magnetic resonance and repeat this after the acute phase. Three quarters of the patients were treated medically. Mortality $(22 \%)$ was lower than the overall rate for posterior fossa hematomas, and the prognosis was better since no survivors had incapacitating sequelae. Conclusion. The specific etiopathogeneses, clinical characteristics and course of bulbar hematomas makes it advisable to do a differential study regarding other types of intracerebral hemorrhage [REV NEUROL 1998; 27: 667-71].

Key words. Angioma cavernosa. Arterial hypertension. Cerebral hemorrhage. Spinal cord.

\section{INTRODUCCIÓN}

Los hematomas de tronco suponen el 5-9\% de las hemorragias intraparenquimatosas y se sitúan habitualmente en la protuberancia. Los hematomas bulbares son en la mayor parte de los casos consecuencia de la extensión hacia ese nivel de hematomas pontinos [1]. La localización bulbar pura es la menos frecuente, cifrándose ésta en un 5\% del total de los hematomas de tronco [2], con lo cual constituyen un $0,25-0,50 \%$ de todos los hematomas intraparenquimatosos. Su diagnóstico y los casos publicados han aumentado en los últimos años debido al empleo sistemático de técnicas de neuroimagen como la TC o RM. Su patogenia en relación con la de las hemorragias intracerebrales en general es distinta, por lo cual también los factores etiológicos que deben considerarse son diferentes. A pesar del mal pronóstico que poseen los hematomas de tronco cerebral, el análisis de los casos de localización exclusivamente bulbar citados en la literatura permite establecer que su evolución a corto y medio plazo es sensiblemente más favorable.

Hemos revisado 18 casos (Tabla I), 3 de ellos propios, en los que la localización bulbar estaba confirmada por pruebas de neuroimagen, hallazgos operatorios o necropsia, valorando su etiología, patogenia, clínica, tratamiento y pronóstico. Quedan, por tanto, excluidos los hematomas bulboprotuberanciales puesto que no es posible asegurar un origen primariamente bulbar.

\section{CASOS CLÍNICOS}

En el Servicio de Neurología de la Ciutat Sanitària i Universitària de Bellvitge (Hospitalet de Llobregat, Barcelona) ingresaron 624 pacientes durante los años 1990 a 1996 con el diagnóstico de hemorragia intraparenquimatosa, de las cuales $3(0,48 \%)$ estaban localizadas en el bulbo.

\section{Recibido: 02.04.98. Aceptado: 04.04.98.}

Servicio de Neurología. Ciutat Sanitària i Universitària de Bellvitge. L'Hospitalet de Ll., Barcelona, España.

Correspondencia: Dr. Mario Jato. Servicio de Neurología. Ciutat Sanitària i Universitària de Bellvitge. Feixa Llarga, s/n. E-08907 L'Hospitalet de Ll., Barcelona.E-mail: mjatod@meditex.es

(C) 1998, REVISTA DE NEUROLOGÍA
Caso 1. Varón de 16 años con voz nasal y diplopía ocasionales de cinco años de evolución. Presentó de forma brusca sialorrea, disfagia, vómitos, diplopía e inestabilidad. La exploración mostraba nistagmus horizontal, paresia de los pares V, VII, IX, X, XI y XII derechos, hipoestesia faciobraquial izquierda, disestesias en pierna y pie izquierdos y dismetría en extremidades derechas. La RM (Fig. 1) mostró una lesión bulbar de 1,8 cm de diámetro en unión bulbomedular derecha, heterogénea, con signos de sangrado reciente sugestiva de cavernoma. La arteriografía reveló lentificación del flujo dentro de la lesión.

El paciente fue intervenido quirúrgicamente, realizándose una craniectomía suboccipital y resección completa de la lesión. En el postoperatorio inmediato presentó bradicardia sinusal y problemas respiratorios y hemodinámicos que motivaron su traslado a la UCI. Posteriormente, la recuperación fue dificultada por varias neumonías aspirativas, resueltas las cuales el enfermo fue dado de alta. En la exploración realizada un año después, se objetivó dificultad para realizar movimientos finos con la mano derecha, hipoestesia táctil y algésica en mano izquierda, paresia de los pares XI y XII derechos y mínima dismetría en extremidades derechas.

Caso 2. Varón de 60 años con antecedentes de HTA, diagnosticada cinco años antes y bien controlada con fármacos, que presentó de manera brusca pérdida de fuerza y parestesias en extremidades derechas, a lo que se añadió un mes después disfagia y disestesias en hemicara derecha. La exploración mostró hemiparesia braquiocrural derecha (4/5), déficit sensitivo en hemicuerpo derecho (incluyendo la cara) para todas las modalidades, hiperestesia palmoplantar derecha, hiperreflexia derecha y reflejo cutaneoplantar derecho indiferente. En la RM se observó un hematoma en la región anterosuperior izquierda del bulbo (Fig. 2). La arteriografía fue normal. El paciente mejoró persistiendo un déficit sensitivomotor discreto en extremidades derechas. Un nuevo estudio mediante RM realizado cinco meses después reveló la existencia de un angioma cavernoso bulbar. En los años posteriores, presentó disfagia y disartria lentamente progresivas.

Caso 3. Mujer de 53 años sin antecedentes de interés que presentó bruscamente cefalea, vómitos, diplopía y sensación de inestabilidad con lateropulsión izquierda. En la exploración se apreció nistagmus rotatorio en la mirada lateral, paresia facial izquierda, disfonía, hipoestesia peribucal izquierda y marcha inestable con lateropulsión izquierda. La RM mostró un hematoma en región posterolateral izquierda del bulbo (Fig. 3). La arteriografía reveló un descenso de la PICA por desplazamiento inferior de la amígdala cerebelosa derecha, sin hallazgos sugestivos de malformación vascular. La evolución fue muy favorable sin que al alta hospitalaria presentara déficit neurológico. 


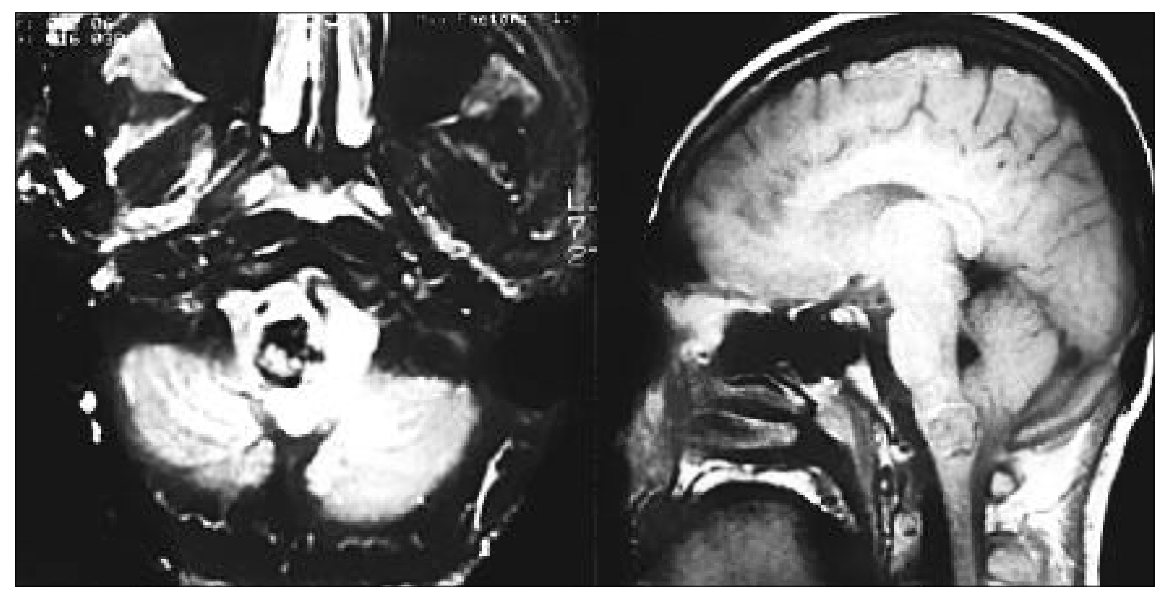

Figura 1. RM (axial $T_{2}$, sagital $T_{1}$ ): cavernoma en unión bulbomedular derecha con signos de sangrado reciente.

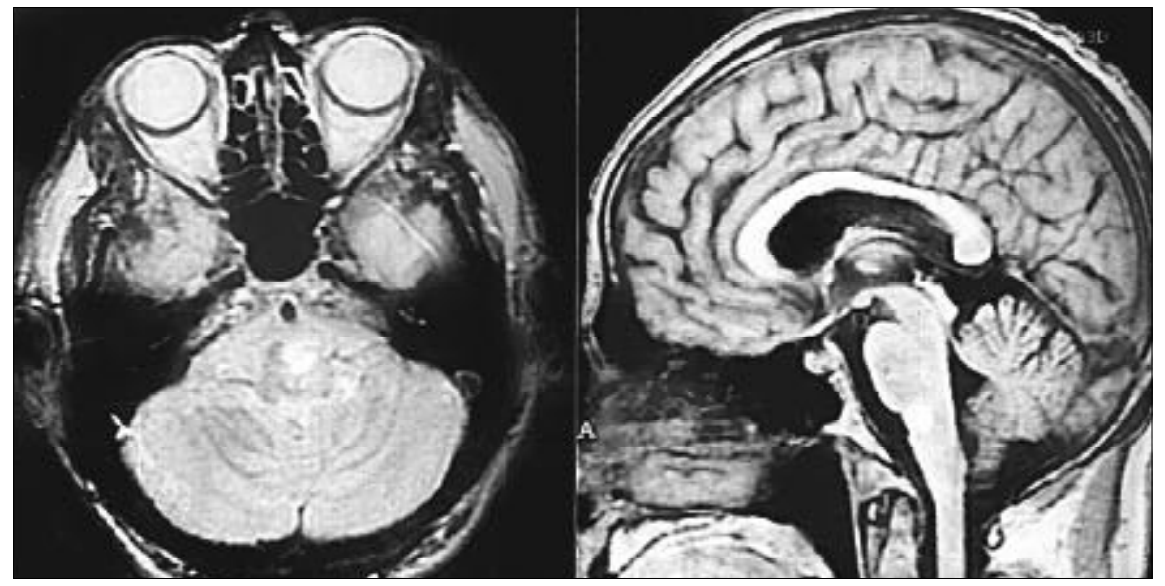

Figura 2. RM (axial DP, sagital $T_{1}$ ): hematoma en región anterosuperior izquierda del bulbo.

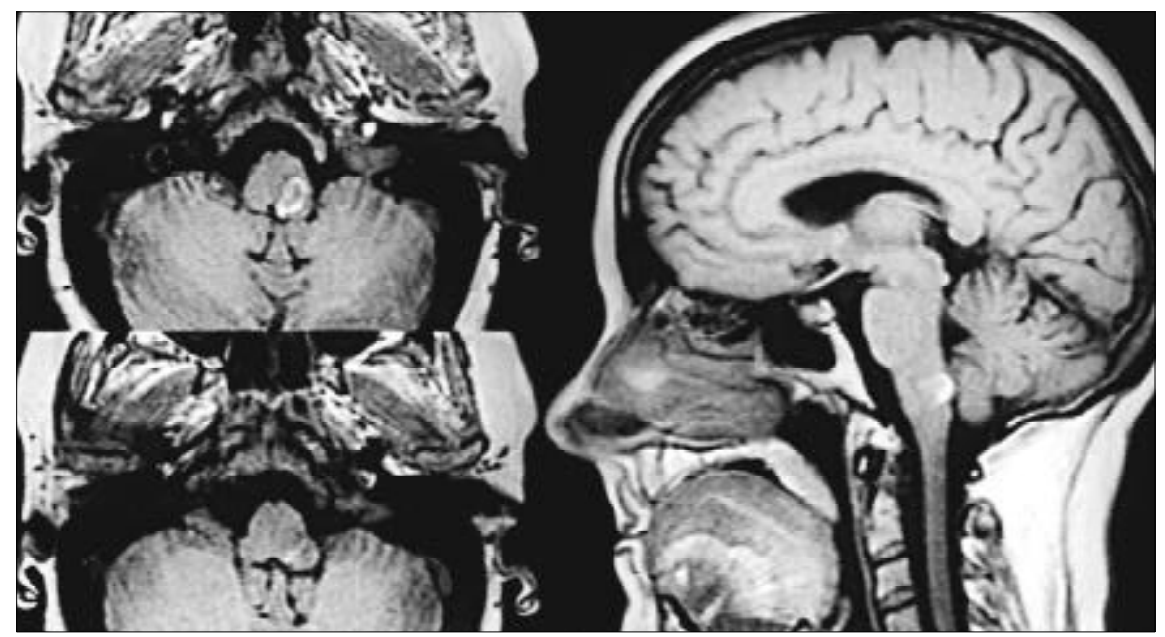

Figura 3. $\mathrm{RM}$ (axial $\mathrm{T}_{1}$, sagital $\mathrm{T}_{1}$ ): hematoma en región posterolateral izquierda del bulbo.
El bulbo tiene unas características anatómicas y hemodinámicas particulares. Así, la mayoría de las arterias bulbares discurren horizontalmente, a diferencia de las localizadas en la protuberancia, cuyo trayecto sigue una curva de concavidad inferior. Se ha sugerido que la cisterna magna podría proteger a la circulación venosa de la compresión entre el bulbo y la base del cráneo, lo que podría explicar lo infrecuente de las hemorragias venosas secundarias bulbares [3]. El estudio histológico del bulbo revela una relativa pobreza vascular en relación con el puente. Además, en el bulbo no existen largas arterias perforantes, como las derivadas de la arteria basilar en la protuberancia, susceptibles de presentar microaneurismas [4], lo que hace que el papel patogénico de la HTA sea menos relevante y que la frecuencia de hemorragias a ese nivel sea sensiblemente menor que en otros lugares del encéfalo como ganglios basales, tálamo o puente [5].

A lo largo de la revisión bibliográfica realizada, el número de varones fue similar al de mujeres (10/8), con una edad media de 42,83 años (16-72, DE 17,96).

La etiología de los hematomas bulbares primarios no es tan amplia como la de las hemorragias intracerebrales(HI) en general; así, se asocian a HTA, tratamiento con anticoagulantes o malformaciones vasculares, pero no hay casos descritos en relación con alcohol y otras drogas, arteritis, angiopatía congófila, tumores o coagulopatías. Aunque la HTA tiene mayor importanciaetiopatogénicaenlas HI $(81 \%)$ [9], de los 18 casos revisados un 39\% tenían antecedentes de HTA (Tabla II). En el $28 \%$ se demostró la existencia de una malformación vascular, cifra muy superior a la señalada para las HI (4\%) [9]; este porcentaje podría ser todavía mayor puesto que diversos autores han sugerido la existencia de malformaciones vasculares crípticas que se destruirían en el momento del sangrado [6], aunque no hay pruebas patológicas de ello. Enunaimportante proporción (39\%) no se encontró causa alguna, lo cual apoya la teoría de que algunos casos se deberían a las mencionadas malformaciones crípticas.

\section{DISCUSIÓN}

Los hematomas de tronco cerebral se sitúan en las tres cuartas partes de los casos en la protuberancia; otro $20 \%$ están localizados en el mesencéfalo, mientras que el $5 \%$ restante afectan de forma primaria al bulbo [2]; mayor porcentaje resultaría si consideráramos la extensión bulbar de hematomas localizados originariamente en la protuberancia.
Los hallazgos clínicos más frecuentes fueron nistagmus, disfagia, parálisis del XII par (quizá por la localización preferentemente posterior de los hematomas), cefalea y déficit sensitivo o motor (Tabla III). Destaca la aparición de parálisis facial (7 pacientes) y diplopía (3 pacientes), atribuibles a extensión rostral del hematoma o a edema periférico a la lesión bulbar y que por este motivo desaparecieron posteriormente. Sólo 2 enfermos sufrieron hipoventilación. 
Tabla I. Hemorragias bulbares espontáneas.

\begin{tabular}{|c|c|c|c|c|c|c|c|}
\hline Autor & Caso & AP & Localización & Arter. & Tratamiento & Evol. & Etiología \\
\hline Kempe [6] & $\vee 25$ & & Posterolateral I & No & Cirugía & Buena & \\
\hline Mastaglia [4] & M 48 & $\begin{array}{l}\text { HTA, } \\
\text { ACO }\end{array}$ & $\begin{array}{l}\text { Posterior I, } \\
\text { medular }\end{array}$ & No & Conservador & Éxitus & \\
\hline Plum y Posner [7] & M 62 & & Medial, medular & No & Conservador & Éxitus & MAV \\
\hline Morel-Maroger [10] & $\vee 56$ & HTA & Posterolateral I & No & Conservador & Buena & \\
\hline Hommel [11] & $\vee 65$ & HTA, DM & Tegmentum D & No & Conservador & Buena & \\
\hline Neumann [12] & V 33 & HTA & $\begin{array}{l}\text { Posterolateral } \\
\text { superior D }\end{array}$ & Normal & $\begin{array}{l}\text { Shunt } \\
\text { ventricular }\end{array}$ & Éxitus & \\
\hline Biller [13] & M 72 & HTA & $\mathrm{D}$ & Normal & Conservador & Buena & \\
\hline Rousseaux [14] & M 21 & & Posteromedial & Normal & Cinugía & Buena & \\
\hline Lee [15] & $\vee 40$ & & Paramedial & Normal & Conservador & Buena & \\
\hline Rousseaux [16] & M 50 & HTA & Anterolateral D & Normal & Conservador & Buena & \\
\hline Barinagarrementería [17] & $\vee 18$ & & Medial I & Normal & Conservador & Buena & \\
\hline Barinagarrementería [17] & $\vee 26$ & Tabaco & $\begin{array}{l}\text { Posteromedial } \\
\text { bilateral }\end{array}$ & Normal & Conservador & Buena & Cavernoma \\
\hline Barinagarrementería [17] & M 22 & & Posteromedial I & Normal & Conservador & Éxitus & \\
\hline Kase [1] & $\vee 54$ & & Posteromedial D & Normal & Conservador & Buena & \\
\hline Wakai [8] & M 50 & & Anterior & Normal & Cirugía & Buena & MAV \\
\hline Caso 1 & $\vee 16$ & & $\begin{array}{l}\text { Unión bulbo- } \\
\text { medular }\end{array}$ & $\begin{array}{l}\text { Flujo } \\
\text { lento }\end{array}$ & Cirugía & Buena & Cavernoma \\
\hline Caso 2 & V 60 & HTA & Anterosuperior I & Normal & Conservador & Buena & Cavernoma \\
\hline Caso 3 & M 53 & & Posterolateral I & Normal & Conservador & Buena & \\
\hline
\end{tabular}

AP: antecedentes; ACO: anticoagulantes orales; V: varón; M: mujer; I: izquierda; D: derecha.

Tabla II. Etiología.

\begin{tabular}{lc}
\hline Desconocida & Casos (\%) \\
\hline HTA & $7(39)$ \\
\hline MAV & $5(28)$ \\
\hline Cavernoma & $2(11)$ \\
\hline Cavemoma + HTA & $2(11)$ \\
\hline ACO + HTA & $1(6)$ \\
\hline
\end{tabular}

Al contrario de lo reseñado en otros trabajos [1], la localización más frecuente es la posterior, que aparece señalada en la mitad de los casos. Su presentación simulando un síndrome bulbar isquémico clásico, como el de Wallenberg, es rara [6], no por lo infrecuente de esa localización, como mencionan algunos autores [1], sino por lo abigarrado de la clínica en caso de hemorragia bulbar.

Es llamativa la escasa rentabilidad de la arteriografía, que fue realizada en 13 de los 18 pacientes $(72 \%)$ sin que mostrara evidencia de malformaciones vasculares en ninguno de ellos. La posibilidad de una malformación vascular subyacente y oculta para las técnicas de neuroimagen en la fase aguda, como en los casos descritos por Wakai [8] y nuestros casos 1 y 2 , hacen aconsejable la repetición de la RM transcurrido un tiempo prudencial después del sangrado aun en el caso de que una primera exploración haya sido normal.

En la mayoría de los enfermos (13/18) se optó por un tratamiento conservador; en 4 se realizó cirugía y en otro fue colocado un shunt ventricular (Tabla IV). La evolución fue buena en 14 de los pacientes, registrándose 4 muertes $(22 \%)$, porcentaje muy inferior a la mortalidad en general de las HI espontáneas $(43,2 \%)$ y específicamente de fosa posterior $(70,9 \%)$ en nuestro medio [9].

De los 14 supervivientes, 3 estaban asintomáticos tras el ictus, y los restantes presentaron secuelas no incapacitantes (Tabla V); las más frecuentes fueron paresia del hipogloso, inestabilidad y dismetría. Destaca el carácter reversible del nistagmus puesto que, a pesar de que es el signo más frecuente (72\%), desaparece en la práctica totalidad de los pacientes y permanece como secuela en sólo uno de ellos. También la disfagia, la parálisis del velo y la disfonía son transitorias, como ya ha sido descrito en otras lesiones vasculares encefálicas unilaterales, probablemente debido a la inervación bilateral de los núcleos implicados [18]. En cuanto a la afectación motora o sensitiva, éstas sólo persistieron en la cuarta parte de los enfermos que la presentaban al ingreso.

El número de casos estudiados es insuficiente para establecer el riesgo de recurrencia, aunque ésta ha sido descrita o al menos sospechada, ya sea en forma ictal o lentamente progresiva, en varios de los pacientes (caso 2) [8,17]. 
Tabla III. Clínica.

\begin{tabular}{|c|c|}
\hline & Casos \\
\hline Nistagmus & 13 \\
\hline Disfagia & 11 \\
\hline Parálisis XII & 11 \\
\hline Cefalea & 9 \\
\hline Hipoestesia extremidades & 9 \\
\hline Hemiparesia & 9 \\
\hline Hipoestesia facial & 8 \\
\hline Ataxia de la marcha & 8 \\
\hline Disfonía & 8 \\
\hline Parestesias/disestesias & 8 \\
\hline Vértigo & 8 \\
\hline Parálisis del velo & 8 \\
\hline Parálisis facial & 7 \\
\hline Disartria & 6 \\
\hline Somnolencia & 5 \\
\hline Inestabilidad & 5 \\
\hline Náuseas/vómitos & 5 \\
\hline Dismetría & 5 \\
\hline Alteración reflejos faríngeos & 5 \\
\hline Horner & 3 \\
\hline Diplopía & 3 \\
\hline Hipoventilación & 2 \\
\hline Hipoacusia & 2 \\
\hline Hipotonía & 1 \\
\hline Rigidez de nuca & 1 \\
\hline Acúfenos & 1 \\
\hline Hipo & 1 \\
\hline Diarrea & 1 \\
\hline Hipohidrosis & 1 \\
\hline Sialorrea & 1 \\
\hline Paresia XI & 1 \\
\hline
\end{tabular}

Tabla IV. Tratamiento

\begin{tabular}{lcc}
\hline & Éxitus & Supervivientes \\
\hline Conservador & 3 & 10 \\
\hline Cirugía & 0 & 4 \\
\hline Shunt ventricular & 1 & 0 \\
\hline
\end{tabular}

Tabla V. Secuelas.

\begin{tabular}{ll}
\hline Paresia XII & 5 \\
\hline Inestabilidad & 4 \\
\hline Asintomáticos & 3 \\
\hline Dismetría & 3 \\
\hline Déficit motor leve & 2 \\
\hline Déficit sensitivo leve & 2 \\
\hline Disartria & 2 \\
\hline Paresia XI & 1 \\
\hline Disfagia & 1 \\
\hline Nistagmus & 1 \\
\hline Parálisis del velo & 1 \\
\hline Disfonía & 1 \\
\hline
\end{tabular}

\section{CONCLUSIONES}

A la vista de los casos publicados y los atendidos en nuestro centro, los hematomas bulbares son cuadros infrecuentes cuya particular etiopatogenia, clínica y evolución exigen un planteamiento diagnóstico y terapéutico diferente en relación con el resto de hematomas intracerebrales o incluso de tronco. Estaría indica$\mathrm{da}$, en general, una actitud conservadora aunque hay que destacar que entre los casos publicados sometidos a cirugía no se ha registrado ningún fallecimiento. La escasa información aportada por la arteriografía hace cuestionable su empleo, siendo preferible la RM en fase aguda y su repetición transcurridos varios meses debido a la posibilidad de malformaciones arteriovenosas angiográficamente ocultas y cavernomas. Probablemente deba valorarse el tratamiento quirúrgico para los pacientes en los que el hematoma se debe a una malformación vascular, y siempre sopesando el tipo de lesión, localización y riesgo de resangrado [19].

\section{BIBLIOGRAFÍA}

1. Kase CS. Midbrain and medullary hemorrhage. In Kase CS, Caplan LR, eds. Intracerebral Hemorrhage. Boston, Mass: Butterworth-Heinemann; 1994. p. 445-63.

2. Le Coz P, Woimant F, George B, et al. Aspects cliniques et évolutifs des hématomes circonscrits du tronc cérébral: apport du scanner $\mathrm{X}$. Rev Neurol (Paris) 1986; 142: 52-60.

3. Mutlu N, Berry RG, Alpers BJ. Massive cerebral hemorrhage: clinical and pathological correlations. Arch Neurol 1963; 8: 644-61.

4. Cole FM, Yates PO. The occurrence and significance of intracerebral micro-aneurysms. J Path Bact 1967; 93: 393-411.

5. Mastaglia FL, Edis B, Kakulas BA. Medullary haemorrhage: a report of two cases. J Neurol Neurosurg Psychiatry 1969; 32: 221-5.

6. Kempe LG. Surgical treatment of an intramedullary haematoma simulating Wallenberg's syndrome. J Neurol Neurosurg Psychiatry 1964; 27: 78-80.
7. Plum F, Posner JB. Diagnosis of stupor and coma. 3 ed. Philadelphia: FA Davis; 1980 . p. 29-30.

8. Wakai S, Okuhata S, Nagai M, et al. Successful removal of an angiographically occult arteriovenous malformation of the ventral medulla. Br J Neurosurg 1990; 4: 429-34.

9. Urtasun F. La hemorragia intracerebral espontánea en Navarra a la luz de la TC. Tesis Doctoral. Facultad de Medicina. Universidad de Navarra, 1987.

10. Morel-Maroger A, Metzger J, Bories J, et al. Les hématomes bénins du tronc cérébral chez les hypertendus artériels. Rev Neurol (Paris) 1982; 138: 437-45.

11. Hommel M, Borgel F, Gaio JM, et al. Latéropulsion ipsilatérale isolée par hématome bulbaire. Rev Neurol (Paris) 1985; 141: 53-4.

12. Neumann PE, Mehler MF, Horoupian DS. Primary medullary hypertensive hemorrhage. Neurology 1985; 35: 925-8. 
13. Biller J, Gentry LR, Adams HP, Morris DC. Spontaneous hemorrhage in the medulla oblongata: clinical MR correlations. J Comput Assist Tomogr 1986; 10: 303-6.

14. Rousseaux M, Griffie G, Dhellemmes P, et al. Hématome bulbaire postéro-médian d'évolution favorable. Étude de la dysautonomie. Rev Neurol (Paris) 1988; 144: 481-8.

15. Lee CC, Ryu SJ. Primary medullary hemorrhage: report of a case. J Formos Med Assoc 1992; 91: 552-4.

\section{HEMATOMAS BULBARES PUROS}

Resumen. Introducción. Los hematomas bulbares puros son poco frecuentes (0,25-0,50 del total de hematomas intraparenquimatosos), probablemente como consecuencia de las particularidades anatómicas y hemodinámicas de la circulación bulbar. Casos clínicos. Hemos seleccionado 18 casos, 3 de ellos propios, en los que la localización exclusivamente bulbar estaba asegurada por la neuroimagen, hallazgos quirúrgicos o necrópsicos. Su aparición se relaciona con HTA, tratamiento anticoagulante y malformaciones vasculares evidentes o crípticas, aunque en un porcentaje destacable (39\%) no se encontró causa alguna. A pesar de la menor importancia de la HTA en su etiopatogenia en relación con otras localizaciones de hemorragia intraparenquimatosa, 7 de los pacientes eran hipertensos. Los hallazgos clínicos más frecuentes fueron nistagmus (72\%), y disfagia y parálisis del hipogloso (61\%). La localización más común fue la posterior. La arteriografía mostró una escasa rentabilidad puesto que fue normal en todos los casos en que se practicó (72\%). La existencia de malformaciones angiográficamente ocultas hace aconsejable la realización de RM y su repetición una vez superada la fase aguda. Las tres cuartas partes de los pacientes recibieron tratamiento médico. La mortalidad (22\%) fue menor que la de los hematomas de fosa posterior en conjunto, y mejor su pronóstico puesto que entre los supervivientes no se registraron secuelas incapacitantes. Conclusión. La peculiar etiopatogenia, clínica y evolución de los hematomas bulbares hace aconsejable un estudio diferenciado respecto a las restantes hemorragias intracerebrales [REV NEUROL 1998; 27: 667-71].

Palabras clave. Angioma cavernoso. Bulbo raquídeo. Hemorragia cerebral. Hipertensión arterial.
16. Rousseaux M, Caron J, Hurtevent JF, et al. Hématome bulbaire dans la région olivaire. Analyse du syndrome autonome. Rev Neurol (Paris) 1993;149:267-273

17. Barinagarrementería F, Cantú C. Primary medullary hemorrhage: report of four cases and review of the literature. Stroke 1994; 25: 1684-7.

18. Gordon C, Hewer RL, Wade DT. Dysphagia in acute stroke. Br Med J 1987; 295: 411-4.

19. Wakai S. Medullary Hemorrhage. Stroke 1995; 26: 706-7.

\section{HEMATOMAS BULBARES PUROS}

Resumo. Introdução. Os hematomas bulbares puros são pouco frequentes (0,25-0,50 do total de hematomas intraparenquimatosos), provavelmente como consequência das particularidades anatómicas e hemodinâmicas da circulação bulbar. Casos clínicos. Seleccionámos 18 casos, 3 deles nos quais a localização exclusivamente bulbar estava assegurada pela neuroimagem, achados cirúrgicos ou necrópsicos. O seu aparecimento relaciona-se com HTA, tratamento anticoagulante e malformações vasculares evidentes ou crípticas, ainda que numa percentagem importante (39\%) não se encontrou causa alguma. Apesar da menor importância da HTA na sua etiopatogenia em relação com outras localizações de hemorragia intraparenquimatosa, 7 dos casos eram doentes hipertensos. Os achados clínicos mais frequentes foram nistagmos (72\%), e disfagia e parésias do hipoglosso (61\%). A localização mais frequente foi a posterior. A arteriografía mostrou uma escassa rentabilidade pois foi normal em todos os casos em que foi efectuada (72\%). A existência de malformações angiograficamente ocultas torna aconselhável a realização de RM e sua repetição após superada a fase aguda. Três quartos dos doentes receberam tratamento médico. A mortalidade (22\%) foi menor que a dos hematomas da fossa posterior em conjunto, e melhor o seu prognóstico visto que entre os sobreviventes não se registaram sequelas incapacitantes. Conclusão. A peculiar etiopatogenia, clínica e evolução dos hematomas bulbares torna aconselhável um estudo diferenciado em relação às restantes hemorragias intracerebrais [REV NEUROL 1998; 27: 667-71].

Palavras chave. Angioma cavernoso. Bulbo raquidiano. Hemorragia cerebral. Hipertensão arterial. 